\title{
$\angle S$ Research Square \\ Repeated Sevoflurane Exposures in Neonatal Rats Increased the Brain Vulnerability to Future Stress Exposure and Resulted in Fear-extinction Deficit
}

\section{Ben-zhen Chen}

West China Hospital,Sichuan University and Sichuan Provincial Women's and Children's Hospital

\section{Li-hua Jiang}

Sichuan Provincial People's Hospital

\section{Ming-qiang Zhang}

Hospital of Chengdu University of Traditional Chinese Medicine,School of Clinical Medicine, Chengdu University of TCM, Chengdu,Sichuan, 610072,China

\section{Wen-qin Zhou}

Sichuan University West China Second University Hospital

\section{Yu-chao Shang}

West China Second University Hospital,Sichuan University

\section{Fang Li}

First University Hospital of West China University: Sichuan University West China Hospital

Bin Liu (D liubinhxyy@163.com )

West China Hospital,Sichuan University https://orcid.org/0000-0001-5503-0053

\section{Research Article}

Keywords: Neonatal sevoflurane, Stress exposure, Neuroapoptosis, Fear extinction, NKCC1/KCC2 ratio

Posted Date: June 18th, 2021

DOI: https://doi.org/10.21203/rs.3.rs-599951/v1

License: (1) (i) This work is licensed under a Creative Commons Attribution 4.0 International License. Read Full License 


\section{Abstract}

Sevoflurane anesthesia during neonatal period was reported to sensitize the rodent animals to stress later in life. The authors tested the hypothesis that repeated sevoflurane exposures in neonatal rats increased the brain vulnerability to future stress exposure and resulted in fear-extinction deficit, and investigated whether the neonatal brain depolarizing $Y$-aminobutyric acid type $A$ receptor $\left(G A B A_{A} R\right)$ is involved in mediating these abnormalities. Neonatal Sprague-Dawley male rats, pretreated with vehicle or the NKCC1 inhibitor, bumetanide, received sequential exposures to $3 \%$ sevoflurane for 2 hours on postnatal days (P) $5,6,7$ and then were exposed to electric foot shock stress in fear conditioning training at P14. Juvenile rats at different developmental brain stage receiving identical sevoflurane exposures on P25, 26, 27 were also studied. The results showed repeated sevoflurane exposures in neonatal rats increased the cationchloride cotransporters NKCC1/KCC2 ratio in the PFC at P14. Repeated exposures to sevoflurane in neonatal rather than juvenile rats enhanced the stress response and exacerbated neuroapoptosis in the PFC after exposed to electric foot shock in fear conditioning training. Neonatal rather than juvenile sevoflurane-exposed rats exhibited deficits in fear extinction training and recall. Pretreatment of neonatal rats prior to sevoflurane exposures with bumetanide reduced the NKCC1/KCC2 ratio at P14 and ameliorated most of the subsequent adverse effects. Our study indicates that repeated sevoflurane exposures in neonatal rats might increase the brain vulnerability to future stress exposure and resulted in fear-extinction deficit, which might be associated with the neonatal enhanced brain depolarizing $G A B A_{A} R$ activity.

\section{Introduction}

Sevoflurane is popularly administered to early life in humans for procedural anesthesia. Neonatal sevoflurane-induced neurocognitive dysfunctions have been demonstrated in many animal studies [1-4]. However, since there are many complex environmental stress and challenges for humans at different physical and physiological stage, the causative links between neonatal sevoflurane exposure and later life neurocognitive dysfunctions were always the subject of vigorous debate.

Dysregulation of the neuroendocrine response to stress were associated with a wide spectrum of stressrelated neuropsychiatric disorders, such as depression and posttraumatic stress disorder (PTSD) [5-7]. People who experience strongly or extremely stress events have an increased risk of PTSD. In recent years, many animal studies have demonstrated neonatal anesthesia could contribute to neuroendocrine dysregulation and sensitize to stress later in life [8-10]. On the other hand, post-anesthesia stress might exacerbate neurodevelopmental deficit for neonatal anesthetic-exposed animals [10]. It was proposed that the adverse developmental results of neonatal anesthesia in animals might be associated with later life experiences [11], it is plausible that neurocognitive abnormalities are the result of a cumulative impact initially programmed by neonatal anesthetic exposure and later aggravated by adverse post-stressful factors such as trauma and various types of psychosocial stress. 
Sevoflurane exposure during the neonatal period of rodent animals was reported to contribute to abnormal social behaviors resembling autism spectrum disorder (ASD) later in life [12]. It has been shown that certain features of ASD symptomatology may predispose the population to a higher risk of trauma exposure and development of PTSD [13-15]. The anesthetic action of sevoflurane include enhancement of $\gamma$-aminobutyric acid (GABA) type $A$ receptor $\left(G A B A_{A} R\right)$ activity. The cation-chloride cotransporters $\mathrm{Na}^{+}-\mathrm{K}^{+}-2 \mathrm{Cl}^{-}$(NKCC1) and $\mathrm{K}^{+}-2 \mathrm{Cl}^{-}-2$ (KCC2) critically regulate neuronal responses to GABA. NKCC1 renders GABA excitatory in immature neurons while expression of KCC2 signals GABA maturation to its inhibitory role. The ontogenetic transition of GABA action from depolarizing to inhibitory occurs on the 2nd postnatal week in the rat brain. Imbalances in NKCC1/KCC2 alter GABA neurotransmission, which may contribute to hyperexcitability and blunted inhibition in neurocircuitry after neonatal exposure to anesthesia. The NKCC1 inhibitor bumetanide could enhance the GABAergic inhibition and alleviated the electrical and behavioral symptoms in models of ASD [16-18]. In the present study, the neonatal sevoflurane-exposed rats were exposed to electric foot shock stress in fear conditioning training and the authors tested the hypothesis that repeated sevoflurane exposures in neonatal rats increased the brain vulnerability to future stress exposure and resulted in fear-extinction deficit, and investigated whether the neonatal brain depolarizing $\mathrm{GABA}_{A} \mathrm{R}$ is involved in mediating these abnormalities.

\section{Methods}

\section{Animals}

The present study was approved by the Ethics Committee of West China Hospital, Sichuan University, and was conducted in accordance with the ARRIVE guidelines and the Guide for the Care and Use of Laboratory Animals from the National Institutes of Health (Bethesda, MD, USA). Pregnant SpragueDawley rats were housed individually in standard conditions with a 12-h light/dark cycle (light from 07:00-19:00) at $24 \pm 1^{\circ} \mathrm{C}$ and ad libitum access to food and water. Five days after the delivery, litters were culled to 12 male pups. Pups were weaned at P21 and housed in groups of four for a further study during juvenile period. To control for litter variability, we used several male pups from different litters for each treatment condition.

\section{Experimental Design Of The Study}

The experimental design of the study is illustrated in Fig. 1. In the first set of experiments (Fig. 1A), the neonatal P5 to P7 male rat pups were randomly assigned to sevoflurane (SEV) and control (CON) groups, then rats were exposed to electric foot shock stress in fear conditioning training at P14. The NKCC1/KCC2 ratio in the prefrontal cortex (PFC) was determined after rats exposed to electric foot shock stress in fear conditioning training. To test the stress response and brain vulnerability to future stress exposure after repeated sevoflurane exposures in neonatal rats, serum corticosterone and neuroapoptosis in the PFC after exposed to electric foot shock stress in fear conditioning training at P14 were measured. To test the hypothesis that repeated sevoflurane exposures in neonatal rats resulted in future fear- 
extinction deficit, fear conditioned-rats were subjected to fear extinction training at P18. Fear extinction recall was assessed at P20. In the second set of experiments (Fig. 1B), in order to test whether sevoflurane exposure at different developmental brain stage contributed to different results, juvenile rats receiving identical sevoflurane exposures on $\mathrm{P} 25,26,27$ were explored.

\section{Neonatal And Juvenile Sevoflurane Exposure}

The neonatal P5 to P7 male rat pups were randomly assigned to sevoflurane (SEV) or control (CON, not exposed to sevoflurane) groups. Rats in the SEV group received 3\% sevoflurane in 02/N2 (fraction of inspired oxygen $50 \%$, or $\mathrm{FiO} 250 \%$ ) for two h daily on three consecutive days from P5 to P7, in a thermostated chamber set to $37 \pm 1^{\circ} \mathrm{C}$. The total gas flow was two Litre $\mathrm{min}^{-1}$. The rats breathed spontaneously, and concentrations of anaesthetic and oxygen were measured continuously using a calibrated Datex side stream analyser that sampled from the interior of the chamber. Anaesthesia with $3 \%$ sevoflurane for two $\mathrm{h}$ does not significantly change blood gas values based on our previous study [11]. After sevoflurane exposure, the rat pups were allowed to recover and were returned to the mothers on gaining the righting reflex. Rat pups in the non-exposed group were separated from the dams for the same duration of time in identical conditions $\left.\left(37 \pm 1^{\circ} \mathrm{C}\right), \mathrm{FiO} 250 \%\right)$, except for exposure to the anaesthetic. To study the role of $G_{A B A} R$ at the time of repeated neonatal sevoflurane exposures, a subgroup of rats received three injections of the $\mathrm{Na}^{+}-\mathrm{K}^{+}-2 \mathrm{Cl}^{-}$cotransporter inhibitor, bumetanide, (BUM, $1.82 \mathrm{mg} / \mathrm{kg}$, intraperitoneally, i.p.) $15 \mathrm{~min}$ prior to initiation of daily sevoflurane exposure (SEV + BUM group). BUM in this concentration/dose range is widely used as the most selective and effective of currently available inhibitors of NKCC1 $[17,18]$. In order to control for the injections of bumetanide prior to anesthesia, other treatment groups received equal volumes of vehicle (VEH, i.p.) saline at P5, P6 and P7. In order to test whether sevoflurane exposure at different developmental brain stage contributed to different results, juvenile rats receiving identical sevoflurane exposures on P25, 26, 27 were explored.

\section{Stress Exposure in Fear Conditioning Training and Fear Extinction Training}

The inescapable electric foot shock stress in fear conditioning training based on the previous study was performed in the present study [19]. Briefly, the conditioning chamber consists of a brightly lit plexiglass box with stainless steel-grid floor (Shanghai Softmaze Information Technology Co. Ltd., Shanghai, China). An infrared video camera mounted on the rear wall of the cabinets recorded the behavior of each rat inside the chamber. The electric foot shock stress in fear conditioning training occurred on P14 for neonatal sevoflurane-exposed rats or on P34 for juvenile sevoflurane-exposed rats. Fear-conditioning experiment consisted of a two min exploration period followed by ten conditioned stimulus (CS)unconditioned stimulus (US) pairings (CS, $70 \mathrm{~dB}$ white noise, 20-s duration; US, $2.0 \mathrm{~mA}$ electric foot shock intensity, 4-s duration; US was delivered during the last 4-s of the CS presentation) separated by one min each. Four days after the conditioning trial, the activities in Open-field test was recorded. The fear conditioning acquisition was determined by receiving 2 min CS presentations in an alternative context with distinct visual and tactile cues after a two min adaptation period and then a fear extinction training 
(for the same set of rats) was performed by receiving $6 \times 2$ min CS presentations (interval of $2 \mathrm{~min}$ ). The rate of the rat's freezing response (defined as the absence of movement except that required for respiration) for every 2 min CS presentation was scored to measure the fear memory. The level of nonspecific freezing provoked by the new context was controlled for two min before the presentation of the cue in that new context. Rats were tested for extinction recall by receiving 2 min CS presentation 48 hrs after extinction training. At the end of testing for each rat, the arena was cleaned with $75 \%$ alcohol to avoid the presence of olfactory cues.

\section{Open-field Test}

The Open-field test was determined at P18 for neonatal sevoflurane-exposed rats and P38 for juvenile sevoflurane-exposed rats. In brief, the rats were placed in the centre of the open-field chamber $(100 \times 100 \times 40 \mathrm{~cm})$. The rat activities were automatically recorded by a video tracking system and were assessed by measuring the total distance travelled during a 10-min session. At the end of testing for each rat, the arena was cleaned with $75 \%$ alcohol to avoid the presence of olfactory cues.

\section{Measurement Of Serum Corticosterone}

Serum corticosterone was measured using commercial ELISA kits (Cayman Chemical Company, Ann Arbor, MI). In order to study the effect of repeated sevoflurane exposures on stress response to electric foot shock in the fear conditioning training, serum levels of corticosterone under basal and stress condition were measured in blood samples collected from the P14 (for neonatal sevoflurane-exposed rats) or P34 (for juvenile sevoflurane-exposed rats) rats 60 min before the process of fear conditioning training and $5 \mathrm{~min}$ after the last unconditioned stimulus (After-US). Blood sampling was done using the "tail clip" method. Specifically, the distal $0.5 \mathrm{~mm}$ of the tail was removed using a sterile scalpel blade, and blood was allowed to drain directly into a microcentrifuge tube.

\section{Western Blot}

Western Blot

The PFC were collected 6 hours after exposed to electric foot shock stress in fear conditioning. Rats were deeply anesthetized with sodium pentobarbital $\left(50 \mathrm{mg} \mathrm{kg}^{-1}\right.$, i.p.) and perfused with phosphate-buffered saline through the left cardiac ventricle. The brains were removed from the skull, put into liquid nitrogen, and then stored at $80^{\circ} \mathrm{C}$ until further use. On the day of analysis, the brain tissue was allowed to equilibrate to a temperature of $4^{\circ} \mathrm{C}$. Western blot analysis was performed as previously described [11]. For primary antibodies, we used rabbit anti-NKCC1 (1:1000; Cell Signaling, USA), anti-KCC2 (1:1000; Cell Signaling, USA), activated Cleaved Caspase-3 (1:500; Cell Signaling, USA). Antibody anti- $\beta$-actin (1:10 000; Sigma, St. Louis, MO) was used to detect $\beta$-actin (42 kDa). The membranes were incubated with horseradish peroxidase-conjugated goat anti-rabbit IgG (1:2000; sc-2004, Santa Cruz Biotechnology, Inc., 
Santa Cruz, CA). The Image J software (NIH Image, Bethesda, MD) was used for semiquantification of the bands. Expression of $\beta$-actin, determined with $\beta$-actin antibody, was used to control for loading differences in the total protein amount.

\section{Statistical Analysis}

Data are presented as mean \pm SEM and analyzed by the Statistical Product for Social Sciences (version 17.0, SPSS Inc., Chicago, Illinois, USA). Normal distribution of data was analyzed using the KolmogorovSmirnov test. Differences among multiple means were assessed by one-way analysis of variance (ANOVA), or repeated-measures two-way ANOVA followed by post hoc Bonferroni test. Independent Student's $t$-test was used for single comparisons between two groups. A $p$ value less than 0.05 was regarded as a statistically significant difference.

\section{Results}

Repeated Sevoflurane Exposures in Neonatal Rats Increased the NKCC1/KCC2 Ratio in the PFC at P14, Which was Alleviated by Pretreated with the NKCC1 Inhibitor Bumetanide

The authors investigated whether repeated sevoflurane exposures in neonatal rats altered the expression the NKCC1/KCC2 ratio in the PFC during the day of fear conditioning training at P14. The results showed repeated sevoflurane exposures in neonatal rats at P5-7 had no statistically difference on expression of

$\operatorname{NKCC1}\left(F_{(2,15)}=1.21, p=0.11\right.$; One-way ANOVA; Fig. 2A) and KCC2 levels in the PFC at P14 $\left(F_{(2,15)}=0.94\right.$, $p=0.08$; One-way ANOVA; Fig. 2B). However, the resulting NKCC1/KCC2 ratio in the PFC at P14 was significantly increased for neonatal sevoflurane-exposed rats $\left(F_{(2,15)}=3.27, p<0.05\right.$; Fig. 2C), which was alleviated by pretreated with the NKCC1 inhibitor bumetanide $\left(\mathrm{F}_{(2,15)}=3.27, p<0.05\right.$; Fig. 2C). These results suggested the possibility the brain depolarizing $G_{A B A} R$ activity might be altered when exposed to the electric foot shock stress in fear conditioning training at P14 for neonatal sevoflurane-exposed rats, which could be attenuated by pretreated with the NKCC1 inhibitor bumetanide.

\section{Repeated Exposures to Sevoflurane in Neonatal Rather Than Juvenile Rats Increased the Stress Response to Electric Foot Shock in the Fear Conditioning Training}

In order to assess whether neonatal exposure to sevoflurane alters the susceptibility to stress exposure, serum corticosterone levels under basal and stress conditions were measured in blood samples collected from P14 rats 60 min before the process of fear conditioning and 5 min after the last unconditioned stimulus (After-US). The results showed neonatal sevoflurane-exposed had unaltered secretion of corticosterone under the basal condition (Fig. 3A), however, the levels of corticosterone under stress condition for neonatal sevoflurane-exposed rats was significantly higher than CON $+\operatorname{VEH}$ group $\left(\mathrm{F}_{(3,21)}=\right.$ 18.17, $p<0.05$, one-way ANOVA; Fig. 3A). Pretreatment of neonatal rats prior to each sevoflurane exposure with BUM significantly attenuated the heightened secretion of corticosterone after the stress exposure in fear conditioning training $\left(\mathrm{F}_{(3,21)}=18.17, p<0.05\right.$; Fig. 3A). 
To test whether sevoflurane exposure at different developmental brain stage affect the stress response, juvenile rats receiving identical sevoflurane exposures on P25, 26, 27 were explored. The results showed there were no statistical difference between SEV + VEH and CON + VEH groups in secretion of corticosterone under basal and stress conditions (Fig. 3B) at P34.

These results indicated repeated exposures to sevoflurane in neonatal rather than juvenile rats increased the neuroendocrine response to future stress exposure, which might be associated with the neonatal enhanced brain depolarizing $\mathrm{GABA}_{\mathrm{A}} \mathrm{R}$ activity.

\section{Repeated Sevoflurane Exposures in Neonatal Rats Increased the Brain Vulnerability to Future Stress Exposure}

The authors tested whether repeated sevoflurane exposures in neonatal rats increased the brain vulnerability to the future stress exposure and whether the adverse results could be attenuated by bumetanide. The basal neuroapoptosis for neonatal sevoflurane-exposed rats were determined without receiving electric foot shock stress at P14. Other independent rat groups were used in determination of stress-induced neuroapoptosis in the PFC six hours after exposed to electric foot shock stress in fear conditioning training at P14. The results showed there was no significantly difference in the neuroapoptosis in the PFC for rats not receiving electric foot shock stress ( $p=0.15$; Fig. 4). However, repeated sevoflurane exposures in neonatal rats at $P 5,6,7$ increased the neuroapoptosis in the PFC six hours after exposed to electric foot shock stress in fear conditioning training at P14 $\left(\mathrm{F}_{(2,15)}=5.12, p<\right.$ 0.01 ; Fig. $3 C$ ), which was alleviated by pretreated with the NKCC1 inhibitor bumetanide (Fig. $3 C, F_{(2,15)}=$ $5.12, p<0.05$; Fig. 3C). Repeated sevoflurane exposures for juvenile rats at P25-27 had no significant effects on neuroapoptosis in the PFC six hours after exposed to stress in fear conditioning training at P34 (Fig. 3D).

These results indicated repeated exposures to sevoflurane in neonatal rather than juvenile rats increased the brain vulnerability to adverse post-stressful factors, which might be associated with the neonatal enhanced brain depolarizing $\mathrm{GABA}_{\mathrm{A}} \mathrm{R}$ activity.

\section{Neonatal Rather Than Juvenile Sevofluraneexposed Rats Exhibited Deficits in Fear Extinction Training and Recall}

For neonatal sevoflurane-exposed rats, there was no significantly difference in the total distance travelled in Open-field test among CON + VEH, SEV + VEH and SEV + BUM groups at P18 (Fig. 5A). The fear conditioning acquisition was determined after the Open-field test. One-way ANOVA analysis revealed there were no group differences in pre-CS freezing prior to CS-presentation $\left(\mathrm{F}_{(2,33)}=0.63, p=0.79\right.$; Fig. 5C). The results showed the rate of freezing under CS-presentation at P18 was significantly increased for CON + VEH, SEV + VEH and SEV + BUM groups (Fig. 5C). Although the SEV + VEH group exhibited higher rate of freezing than $\mathrm{CON}+\mathrm{VEH}$ and SEV + BUM groups in fear conditioning acquisition, there was no statistical difference among CON + VEH, SEV + VEH and SEV + BUM groups $\left(\mathrm{F}_{(2,33)}=0.86, p=0.13\right.$; 
Fig. 5C; One-way ANOVA). For juvenile sevoflurane-exposed rats, there was no significantly difference in the total distance travelled in Open-field test between CON and SEV groups at P38 (Fig. 5B). The fear conditioning acquisition at P38 was significantly increased for CON and SEV groups at P38 (Fig. 5D).

Following the determination of fear conditioning acquisition, the authors performed the fear extinction training for neonatal sevoflurane-exposed rats, which was the laboratory basis of exposure therapy for anxiety disorders. Regarding the rate of freezing change during extinction training, there was a significant effect of extinction trial $\left(F_{(5,165)}=24.43, p<0.01\right.$; Fig. 6A). Repeated measures two-way ANOVA showed there was significant effect of treatment $\left(\mathrm{F}_{(2,165)}=18.87, p<0.01\right.$; Fig. $\left.6 \mathrm{~A}\right)$ on the rate of freezing response in fear-extinction training and an interaction of treatment-by-trial $\left(F_{(10,165)}=29.65, p<0.01\right.$; Fig. 6A). The post hoc Bonferroni test showed SEV + VEH rats had a higher rate of freezing response than the $\mathrm{CON}+\mathrm{VEH}$ rats at CS3, CS4, CS5 and CS6. Pretreated with the NKCC1 inhibitor bumetanide before neonatal sevoflurane exposures lowered the rate of freezing response in fear-extinction training compared with SEV + VEH rats at CS5 and CS6.

The CON + VEH and SEV + BUM rats exhibited comparable, low CS-elicited freezing at CS5 and CS6 in fear-extinction training, which suggested the success in retention of fear extinction for both groups (Fig. 6A), however, SEV + VEH rats still exhibited high CS-elicited freezing compared with the CON + VEH and SEV + VEH groups at CS5 and CS6, suggesting the deficit in retention of fear extinction. Then rats were tested for extinction recall at P20 by receiving 2 min CS presentation. All groups exhibited comparable, low, pre-CS freezing during the adaptation period prior to extinction recall $\left(\mathrm{F}_{(2,33)}=1.28, p=\right.$ 0.78 ; Fig. 6B ), however the groups differed significantly in CS-elicited freezing $\left(F_{(2,33)}=16.21, p<0.01\right.$; Fig. 6B). The post hoc Bonferroni test showed that the SEV + VEH rats exhibited significantly higher rate of freezing response compared with the CON + VEH group $\left(\mathrm{F}_{(2,33)}=16.21, p<0.01\right.$; Fig. 6B) in extinction recall. Pretreated with the NKCC1 inhibitor bumetanide before neonatal sevoflurane exposures reduced the rate of freezing response in fear-extinction recall $\left(F_{(2,33)}=16.21, p=0.019\right.$; Fig. 6B).

For juvenile sevoflurane-exposed rats, there was no difference between the two groups in pre-CS freezing prior to extinction training ( $p=0.78$; Fig. $6 \mathrm{C})$. Regarding the rate of freezing change during extinction training, there was significant effect of extinction trial $\left(F_{(5,110)}=16.23, p<0.01 ;\right.$ Fig. $\left.6 C\right)$. However, repeated measures two-way ANOVA showed there was no effect of group $\left(\mathrm{F}_{(1,110)}=0.96, p=0.67 ; \mathrm{Fig} .6 \mathrm{C}\right)$ and trial-by-group interaction $\left(F_{(5,110)}=1.49, p=0.39\right.$; Fig. $\left.6 \mathrm{C}\right)$, indicating that both groups exhibited comparable rates of extinction (Fig. $6 \mathrm{C}$ ). Both groups exhibited comparable, low, pre-CS freezing during the adaptation period prior to extinction recall $(p=0.57$; Fig. 6D). There was also no difference between the two groups in CS-elicited freezing (Fig. 6D).

\section{Discussion}

The present study tested the hypothesis that repeated sevoflurane exposures in neonatal rats increased the brain vulnerability to future stress exposure and resulted in fear-extinction deficit, and investigated 
whether the neonatal brain depolarizing $\mathrm{GABA}_{A} \mathrm{R}$ was involved in mediating these abnormalities. The results showed that repeated sevoflurane exposures in neonatal rats increased the NKCC1/KCC2 ratio in the PFC at P14. Repeated exposures to sevoflurane in neonatal rather than juvenile rats increased the stress response and exacerbated neuroapoptosis in the PFC after exposed to electric foot shock stress in fear conditioning training. Neonatal sevoflurane-exposed rats exhibited deficits in the fear extinction training and recall. Pretreatment of neonatal rats prior to sevoflurane exposures with bumetanide reduced the NKCC1/KCC2 ratio during the stress exposure in the fear conditioning training and ameliorated most of the subsequent adverse effects. Our study indicate that repeated sevoflurane exposures in neonatal rats increased the brain vulnerability to future stress exposure and resulted in fear-extinction deficit, which might be associated with the neonatal enhanced brain depolarizing $G_{A B A_{A}} R$ activity.

During early development, neocortical neurons undergo activity-dependent, caspase-3-mediated apoptosis. Programmed cell death is an essential process for the proper development of the nervous system, which could be triggered by physiological and pathological stimuli. In the immature brain during a period of rapid development, neuronal apoptosis could be induced by excessive activation of $G A B A_{A} R$ [20]. There is a high expression of caspase-3 in the neocortex during the early postnatal period and a subsequent decrease to low levels in the adult. In the present study, there is no significantly difference in the neuroapoptosis in the PFC for rats not exposed to electric foot stress between CON and SEV groups, however, repeated exposures to sevoflurane in neonatal rats increased the neuroapoptosis in the PFC six hours after exposed to electric foot shock stress in fear conditioning training at P14, suggesting the increased susceptibility of brain to stress-induced brain injury for neonatal sevoflurane-exposed rats. Repeated sevoflurane exposures in neonatal rats might contribute to disturbance in brain network activity and interfere with the activity-dependent regulation of developmental apoptosis, which eventually increased the brain vulnerability to future stress exposure and resulted in neurological dysfunction.

Disturbing NKCC1/KCC2 balance was associated with brain circuit hyperexcitability and contributed to a wide spectrum of stress-related neurocognitive and psychosocial dysfunctions [21-25]. The peak expression of NKCC1 in rodents is around P5-P7 [18], which provide the driving force for depolarizing $\mathrm{GABA}_{A} \mathrm{R}$-mediated responses in immature neurons $[18,22,26]$. NKCC1 was reported to play a critical role in regulating the function of brain GABAergic transmission and modulating the vulnerability to maternal separation-induced susceptibility to later life stress [27]. Since $G A B A_{A} R$-mediated inhibition of the limbichypothalamic-pituitary-adrenal (LHPA) axis activity was an crucial mechanism of adaption to psychosocial stress in the mature brain [28, 29], the GABA-based mechanism of adaptation to psychosocial stress in the mature brain might lead to a opposite, stress-exacerbating effect in the neonatal brain. In the present study, the NKCC1/KCC2 ratio was increased in the PFC for neonatal sevoflurane-exposed rats $\mathrm{P} 14$, the neuroendocrine response and brain vulnerability to stress exposure in the fear conditioning training was significantly increased for neonatal sevoflurane-exposed rats, which was alleviated by administration of NKCC1 inhibitor bumetanide before neonatal sevoflurane exposures. We proposed the mechanism that the enhanced depolarizing $G_{A B A} R$ activity after neonatal repeated sevoflurane exposures at P5-P7 contributed to the dysregulation of the neuroendocrine response and 
increased the brain vulnerability to stress exposure later in life. The alleviating effects of bumetanide on neuroendocrine dysregulation for neonatal sevoflurane-exposed rats is that bumetanide, by reversing GABA-initiated signaling from depolarizing to inhibitory, facilitates adaptation of the neonatal brain to environmental stressful factors later in life.

Fear extinction is the experimental basis of fear inhibition, the common psychological therapy of anxiety disorders. The anxiety in fear extinction are characterized by impairment in the ability to inhibit fear. Fear extinction involves reciprocal PFC interactions with thalamic, hippocampal, and neocortical pathways [30]. Recall of fear extinction, which is thought to aid in recovery from a psychologically traumatic event, is hypothesized to be deficient in PTSD. In the current study, repeated exposures to sevoflurane in neonatal rather than juvenile rats resulted in heightened stress response in the fear condition training, and exacerbated the deficits in fear extinction training and recall, thus the authors proposed the possibility repeated sevoflurane exposures in neonatal rats predisposed them to development of PTSD. Repeated exposures to sevoflurane in neonatal rats might predispose them to a higher risk of stress exposure and development of neurocognitive dysfunctions later in life. Since the ontogenetic transition of GABA action from depolarizing to inhibitory occurs on the 2nd postnatal week in the rat brain [31,32], the aggravating effects of sevoflurane exposure in neonatal rather than juvenile rats in fear extinction training and recall might be associated with paradoxical hyperexcitatory $G_{A B A} R$ activity during neonatal life .

The causative links between neonatal sevoflurane exposure and later life neurocognitive dysfunctions were always the subject of vigorous debate. In the present study, our findings indicated that repeated sevoflurane exposures in neonatal rats increased the brain vulnerability to future stress exposure and resulted in fear-extinction deficit, which might be associated with the neonatal enhanced brain depolarizing $G_{A B A} R$ activity. The present findings alert the increased risk for adverse post-stressful factors after subject to early life sevoflurane exposure and suggest a new potential target in prevention of anesthesia-related neurocognitive dysfunctions. More importantly, future research should carefully explore the etiological relationship between the stressful factors later in life and sevoflurane-induced neurocognitive dysfunctions.

\section{Declarations}

\section{Authors' Contributions}

Study design: Bin Liu, Ben-zhen Chen.

Experimentation: Wen-qin Zhou, Yu-chao Shang, Fang Li.

Data analysis: Ming-qiang Zhang, Li-hua Jiang.

Overall project supervision: Bin Liu.

Final manuscript preparation: Ben-zhen Chen. 
Acknowledgements

We thank Bi Lei for technical assistance.

\section{Funding}

This work was supported by the grants from the National Natural Science Foundation of China (Nos. 81701090) and Key research project of Health Commission of Sichuan Province (Grant Number: 19ZD009).

\section{Availability of Data and Material}

The data that support the findings of this study are available from the corresponding author, Bin Liu, upon reasonable request.

\section{Compliance with Ethical Standards}

\section{Ethics Approval}

The present study was approved by the Ethics Committee of West China Hospital, Sichuan University, and was conducted in accordance with the ARRIVE guidelines and the Guide for the Care and Use of Laboratory Animals from the National Institutes of Health (Bethesda, MD, USA).

\section{Conflicts of interest}

The authors declare that they have no conflicts of interest.

\section{Consent to Participate}

Not applicable.

\section{Consent for Publication}

All authors have seen and approved the manuscript to be published and contributed intellectually to this work.

\section{References}

1. Yu Y, Yang $Y$, Tan $\mathrm{H}$ et al (2020) Tau contributes to sevoflurane-induced neurocognitive impairment in neonatal mice. Anesthesiology 133:595-610

2. Yu X, Zhang F, Jinshan Shi J (2018) Neonatal exposure to sevoflurane caused cognitive deficits by dysregulating SK2 channels and GluA2-lacking AMPA receptors in juvenile rat hippocampus. Neuropharmacology 141:66-75 
3. Ji M, Wang Z, Sun X et al (2017) Repeated neonatal sevoflurane exposure-induced developmental delays of parvalbumin interneurons and cognitive impairments are reversed by environmental enrichment. Mol Neurobiol 54:3759-3770

4. Sun Z, Satomoto M, Adachi YU, Kinoshita H, Makita K (2016) Inhibiting NADPH oxidase protects against long-term memory impairment induced by neonatal sevoflurane exposure in mice. $\mathrm{Br} \mathrm{J}$ Anaesth 117:80-86

5. Ménard C, Pfau ML, Hodes GE, Russo SJ (2017) Immune and neuroendocrine mechanisms of stress vulnerability and resilience. Neuropsychophar 42:62-80

6. Molaie AM, Maguire J (2018) Neuroendocrine abnormalities following traumatic brain injury: an important contributor to neuropsychiatric sequelae. Front Endocrinol 9:176

7. DePierro J, Lepow L, Feder A, Yehuda R (2019) Translating molecular and neuroendocrine findings in posttraumatic stress disorder and resilience to novel therapies. Biol Psychiatry 86:454-463

8. Tan S, Xu C, Zhu W et al (2014) Endocrine and neurobehavioral abnormalities induced by propofol administered to neonatal rats. Anesthesiology 121:1010-1017

9. Xu C, Tan S, Zhang J et al (2015) Neonatal anesthesia with sevoflurane: developmental neuroendocrine abnormalities and alleviating effects of the corticosteroid and $\mathrm{Cl}^{-}$importer antagonists. Psychoneuroendocrino 60:173-181

10. Ju L, Yang J, Gravenstein $\mathrm{N}$ et al (2017) Role of environmental stressors in determining the developmental outcome of neonatal anesthesia. Psychoneuroendocrino 81:96-104

11. Zhang M, Ji M, Zhao Q et al (2015) Neurobehavioural abnormalities induced by repeated exposure of neonatal rats to sevoflurane can be aggravated by social isolation and enrichment deprivation initiated after exposure to the anaesthetic. $\mathrm{Br} J$ Anaesth 115:752-760

12. Satomoto M, Satoh Y, Terui K et al (2009) Neonatal exposure to sevoflurane induces abnormal social behaviors and deficits in fear conditioning in mice. Anesthesiology 110:628-637

13. Kerns CM, Newschaffer CJ, Berkowitz SJ (2015) Traumatic childhood events and autism spectrum disorder. J Autism Dev Disord 45:3475-3486

14. Hoover DW (2015) The Effects of Psychological Trauma on Children with Autism Spectrum Disorders: a Research Review. Review Journal of Autism Developmental Disorders 2:287-299

15. Haruvi-Lamdan N, Horesh D, Golan O (2018) PTSD and autism spectrum disorder: co-morbidity, gaps in research, and potential shared mechanisms. Psychol Trauma 10:290-299

16. Lemonnier E, Villeneuve N, Sonie S et al (2017) Effects of bumetanide on neurobehavioral function in children and adolescents with autism spectrum disorders. Transl Psychiatry 7:e1056

17. Tyzio R, Nardou R, Ferrari DC et al (2014) Oxytocin-mediated GABA inhibition during delivery attenuates autism pathogenesis in rodent offspring. Science 343:675-679

18. Dzhala VI, Talos DM, Sdrulla DA et al (2005) NKCC1 transporter facilitates seizures in the developing brain. Nat Med 11:1205-1213 
19. Quirk GJ, Garcia R, González-Lima F et al (2006) Prefrontal mechanisms in extinction of conditioned fear. Biol Psychiatry 60:337-343

20. Ikonomidou C, Bittigau P, Koch C et al (2001) Neurotransmitters and apoptosis in the developing brain. Biochem Pharmacol 62:401-405

21. Liu R, Wang J, Liang S, Zhang G, Yang X (2020) Role of NKCC1 and KCC2 in epilepsy: from expression to function. Front Neurol 10:1407

22. Ben-Ari Y (2017) NKCC1 chloride importer antagonists attenuate many neurological and psychiatric disorders. Trends Neurosci 40:536-554

23. Yang J, Ju L, Yang C, et al ( (2018) Effects of combined brief etomidate anesthesia and postnatal stress on amygdala expression of $\mathrm{Cl}^{-}$cotransporters and corticotropin-releasing hormone and alcohol intake in adult rats. Neurosci Lett 685:83-83 9)

24. Ju L, Yang L, Morey TE, et al ( (2018) Role of epigenetic mechanisms in transmitting the effects of neonatal sevoflurane exposure to the next generation of male, but not female, rats. Br J Anaesth 121:406-406 16)

25. Cabrera OH, Tesic V, Tat QL et al (2020) Sevoflurane-induced dysregulation of cation-chloride cotransporters NKCC1 and KCC2 in neonatal mouse brain. Mol Neurobiol 57:1-10

26. Pfeffer CK, Stein V, Keating DJ et al (2009) NKCC1-dependent GABAergic excitation drives synaptic network maturation during early hippocampal development. J Neurosci 29:3419-3430

27. Hu D, Yu Z, Zhang Y et al (2017) Bumetanide treatment during early development rescues maternal separation-induced susceptibility to stress. Sci Rep 7:11878

28. Mody I, Maguire J (2012) The reciprocal regulation of stress hormones and GABA(A) receptors. Front Cell Neurosci 6:4

29. Crowley SK, Girdler SS (2014) Neurosteroid, GABAergic and hypothalamic pituitary adrenal (HPA) axis regulation: what is the current state of knowledge in humans? Psychopharmacology 231:36193634

30. Quirk GJ, Garcia R, González-Lima F (2006) Prefrontal mechanisms in extinction of conditioned fear. Biol Psychiatry 60:337-343

31. Khazipov R, Khalilov I, Tyzio R et al (2004) Developmental changes in GABAergic actions and seizure susceptibility in the rat hippocampus. Eur J Neurosci 19:590-600

32. Glykys J, Dzhala VI, Kuchibhotla KV et al (2009) Differences in cortical versus subcortical GABAergic signaling: a candidate mechanism of electroclinical uncoupling of neonatal seizures. Neuron 63:657-672

\section{Figures}


Sevoflurane exposure at different developmental brain stage

Stress exposure in

\begin{tabular}{lccccc} 
A & Birth & CON/SEV & fear-conditioning training & Fear extinction training & Test for extinction recall \\
\hline P0 & P5-7 & P14 & P18 & P20
\end{tabular}

Stress exposure in

\begin{tabular}{ccccc} 
B & CON/SEV & fear-conditioning training & Fear extinction training & Test for extinction recall \\
\cline { 2 - 4 } & P25-27 & P34 & P38 & P40
\end{tabular}

Figure 1

Experimental design of the study.
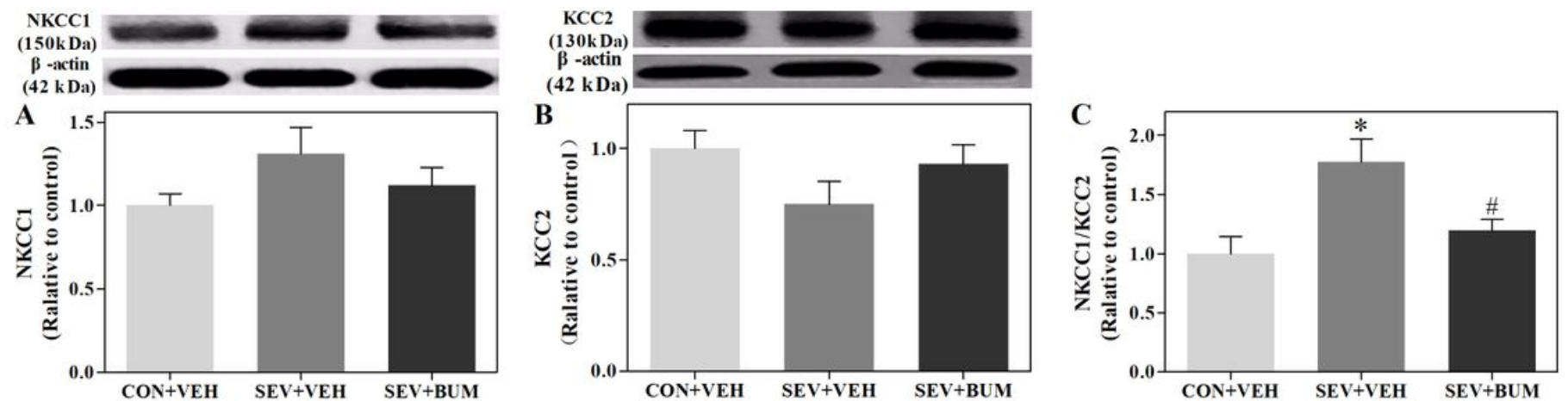

Figure 2

Repeated sevoflurane exposures in neonatal rats at P5-7 had no statistically difference on expressions of NKCC1 (A) and KCC2 (B) levels in the PFC at P14. However, the resulting NKCC1/KCC2 ratio in the PFC at P14 was significantly increased for neonatal sevoflurane-exposed rats (C), which was alleviated by pretreated with the NKCC1 inhibitor bumetanide (C). (mean \pm SEM; $n=6$ rats/group; *p $\leq 0.05$ vs. CON + VEH group; $\# \mathrm{p} \leq 0.05$ vs. SEV + VEH group, One-way ANOVA) 

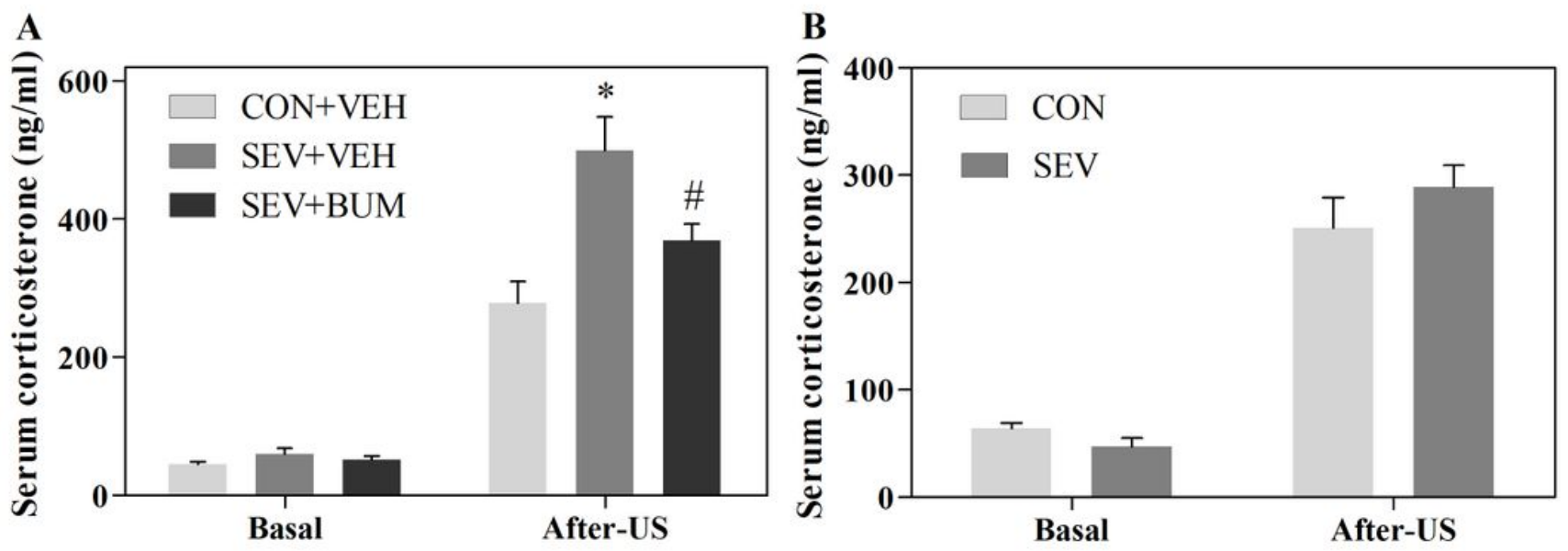

C
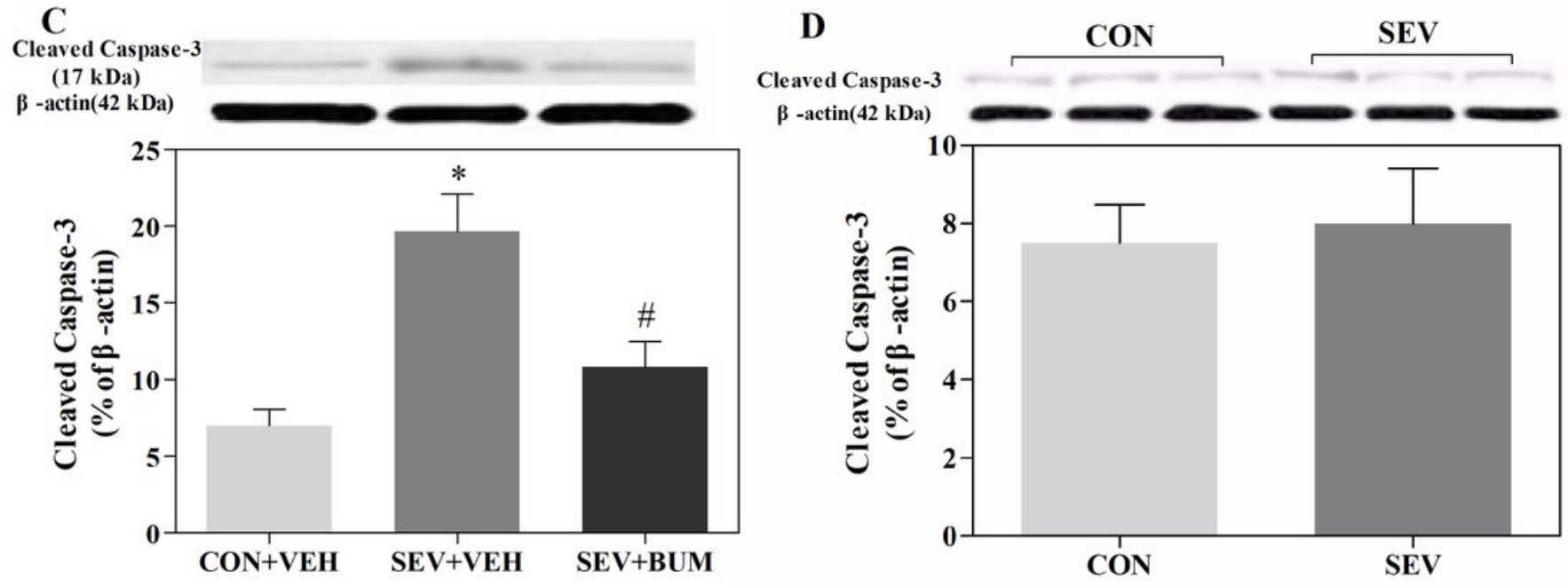

Figure 3

Repeated sevoflurane exposure in neonatal rather than juvenile rats enhanced the secretion of corticosterone after exposed to electric foot shock stress in fear conditioning training. Serum levels of corticosterone under basal and stress condition were measured from blood samples of neonatal $(A)$ and juvenile (B) sevoflurane-exposed rats. (C) Repeated sevoflurane exposures in neonatal rats at P5, 6, 7 increased the neuroapoptosis in the PFC six hours after exposed to electric foot shock stress in fear conditioning training at P14, which was alleviated by pretreated with the NKCC1 inhibitor bumetanide. (D) Repeated sevoflurane exposures for juvenile rats at P25-27 had no significant effects on neuroapoptosis in the PFC six hours after exposed to stress in fear conditioning training at P34. (mean \pm SEM; $n=6$ rats/group; ${ }^{*} p \leq 0.05$ vs. CON + VEH group; $\# p \leq 0.05$ vs. SEV + VEH group, One-way ANOVA) 


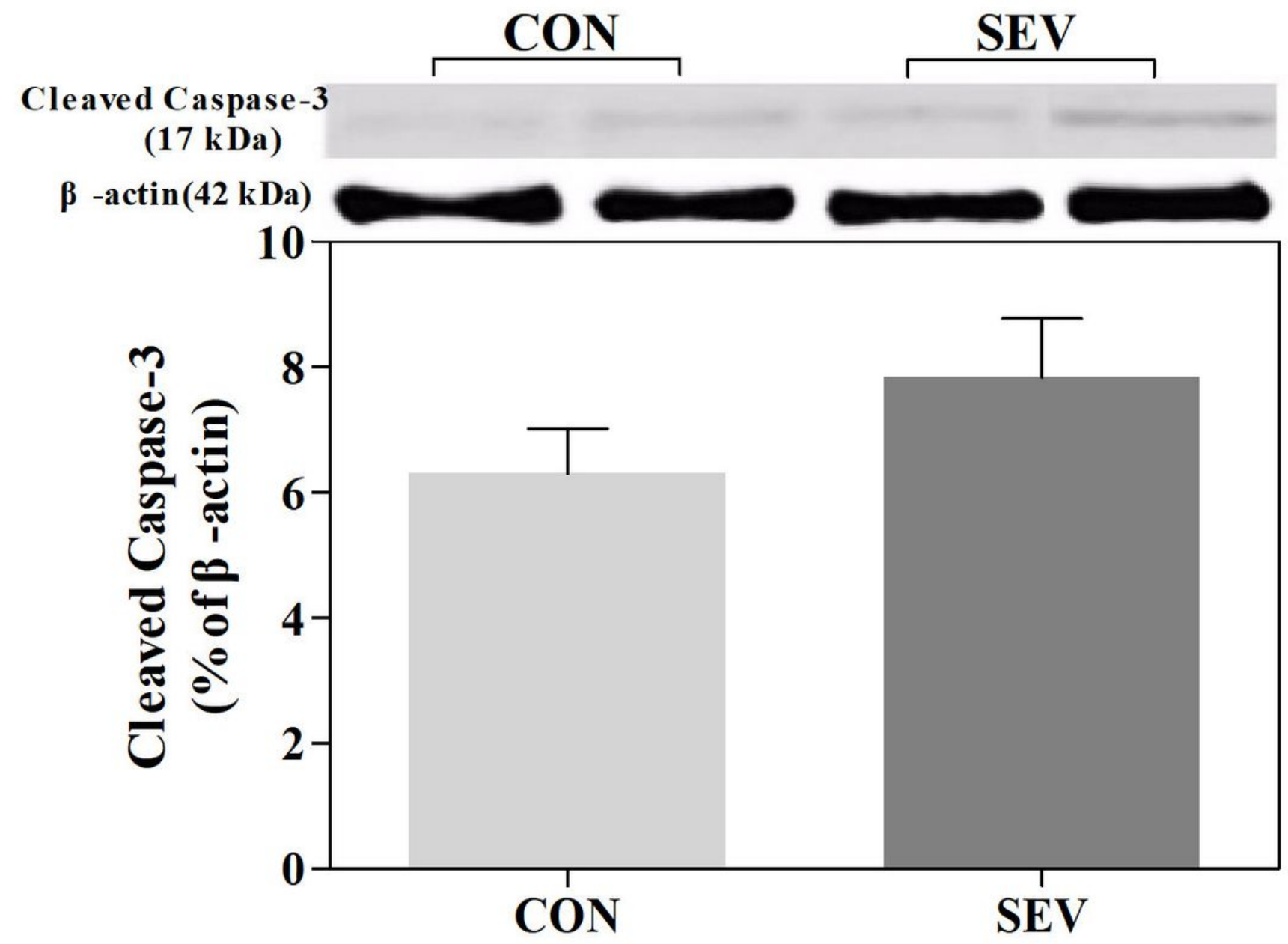

Figure 4

There was no statistical difference in the expression of activated cleaved caspase- 3 in the PFC for rats not exposed to electric foot shock stress in fear conditioning training at P14. (mean \pm SEM; $n=6$ rats/group; Student's t test) 

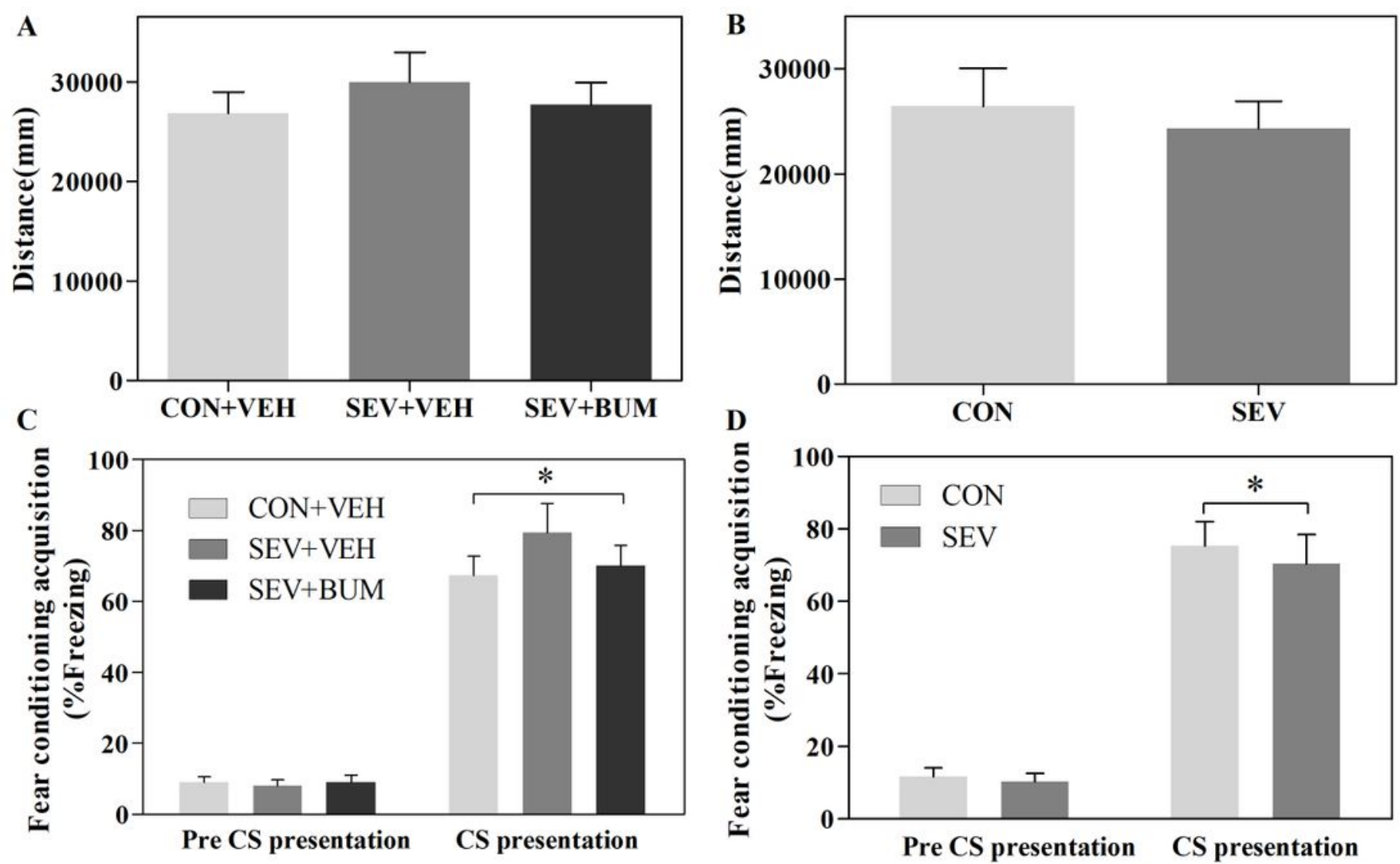

Figure 5

The activity in the Open-field test and the fear conditioning acquisition (determined by receiving 2 min CS presentation) were determined at P18 for neonatal ( $A$ and $C$ ) and P38 for juvenile (B and D) sevofluraneexposed rats. (mean $\pm \mathrm{SEM} ; \mathrm{n}=12$ rats/group; ${ }^{\star} \mathrm{p} \leq 0.05$ vs. rats without receiving CS presentation) 

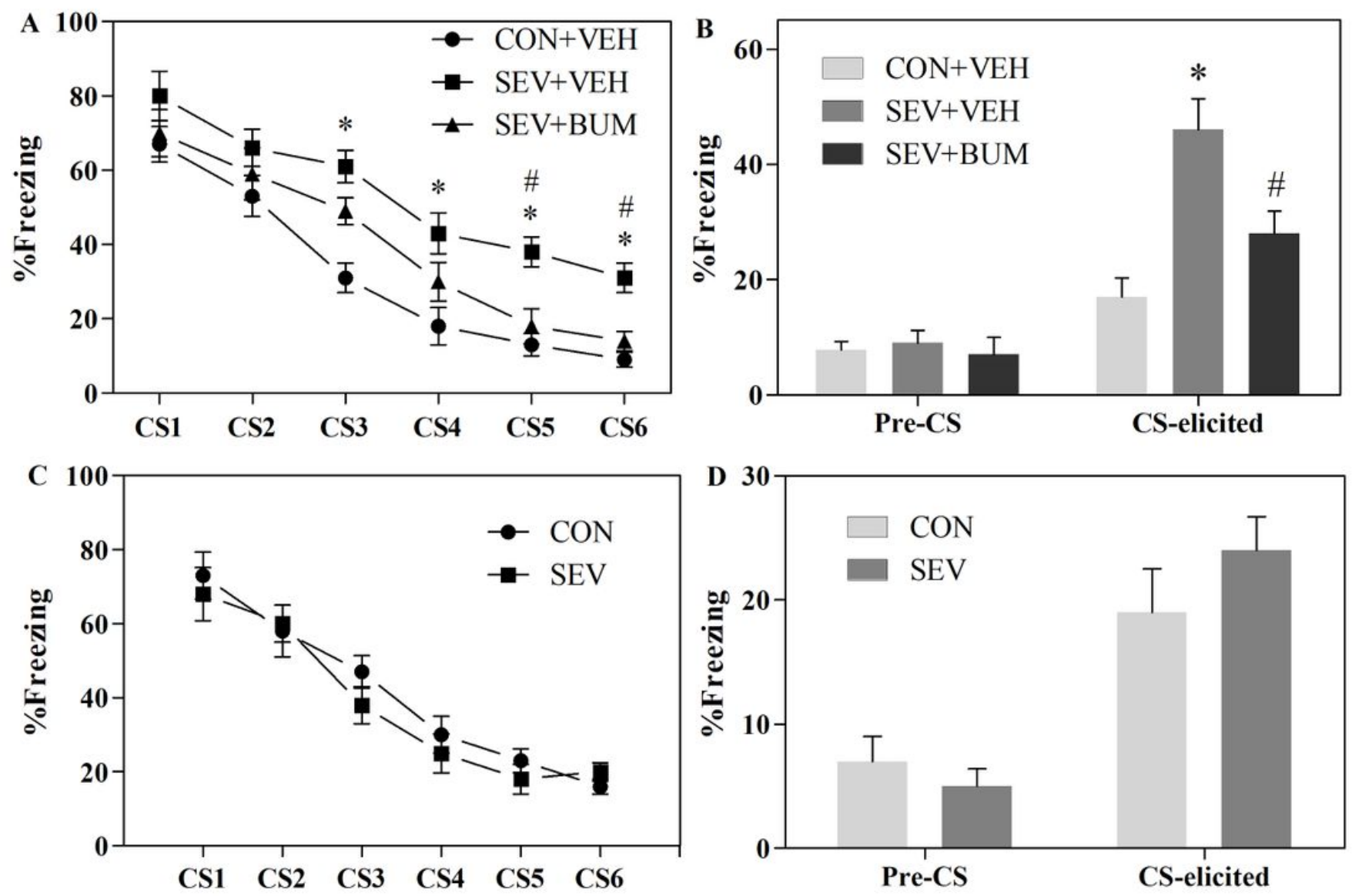

Figure 6

Repeated sevoflurane exposure in neonatal rather than juvenile rats exacerbated the deficits in fear extinction training and recall. The rate of freezing response for every 2 min CS presentation was scored at P18 for neonatal (A) and P38 for juvenile (C) sevoflurane-exposed rats to distinguish the fear memory during extinction training. (mean $\pm \mathrm{SEM} ; \mathrm{n}=12$ rats/group; ${ }^{\star} p \leq 0.05$ vs. CON $+\mathrm{VEH}$ group at corresponding CS presentation; $\# p \leq 0.05$ vs. SEV + VEH group at corresponding CS presentation, Twoway repeated measures ANOVA). The extinction recall was tested for neonatal (B) and juvenile (D) sevoflurane-exposed rats by receiving $2 \mathrm{~min} C S$ presentation $48 \mathrm{hr}$ after the extinction training. (mean \pm $\mathrm{SEM} ; \mathrm{n}=12$ rats/group; ${ }^{\star} p \leq 0.05$ vs. CON + VEH group; $\# p \leq 0.05$ vs. SEV + VEH group, One-way ANOVA) 